\title{
Utilization of various lignocellulosic biomass for the production of nanocellulose: a comparative study
}

\author{
B. Deepa $\cdot$ Eldho Abraham $\cdot$ Nereida Cordeiro • \\ Miran Mozetic • Aji P. Mathew · Kristiina Oksman • \\ Marisa Faria $\cdot$ Sabu Thomas $\cdot$ Laly A. Pothan
}

Received: 12 August 2014/Accepted: 19 January 2015/Published online: 28 January 2015

(C) Springer Science+Business Media Dordrecht 2015

\begin{abstract}
Nanocellulose was successfully extracted from five different lignocellulosic biomass sources viz. banana rachis, sisal, kapok, pineapple leaf and coir using a combination of chemical treatments such as alkaline treatment, bleaching and acid hydrolysis. The shape, size and surface properties of the nanocellulose generally depend on the source and hydrolysis conditions. A comparative study of the fundamental properties of raw material, bleached and nanocellulose was carried out by means of Fourier transform infrared spectroscopy, scanning electron microscopy, atomic force microscopy, transmission electron microscopy, birefringence, X-ray diffraction, inverse gas chromatography and thermogravimetric analysis. Through the characterization of the nanocellulose obtained from different sources, the isolated nanocellulose showed an average diameter in the range of 10-25 nm, high crystallinity, high thermal stability and a great potential to be used with acid coupling agents due to
\end{abstract}

B. Deepa · L. A. Pothan $(\bowtie)$

Department of Chemistry, Bishop Moore College, Mavelikara 690101, Kerala, India

e-mail: lalybmc@gmail.com; lapothan@gmail.com

B. Deepa · A. P. Mathew · K. Oksman

Division of Materials Science, Department of Engineering

Sciences and Mathematics, Luleå University of

Technology, 97187 Luleå, Sweden

B. Deepa $\cdot$ S. Thomas $\cdot$ L. A. Pothan

Department of Chemistry, C.M.S. College,

Kottayam 686001, Kerala, India a predominantly basic surface. This work provides an insight into the effective utilization of a variety of plant biomass as a potential source for nanocellulose extraction.

Keywords Nanocellulose - Biomass ·

Thermochemical modification - Cellulose nanofibrils . Surface characteristics

\section{Introduction}

The production of innovative materials from renewable and abundant bioresources, such as nanocelluloses obtained from cellulosic sources, is becoming an important area of research since it offers a unique combination of high physical properties producing a variety of high-value products with low impact on the environment (Xu et al. 2013; Kengkhetkit and Amornsakchai 2014; Hossain et al. 2014; Maiti et al.

E. Abraham

Robert H Smith Faculty of Agriculture, Food and

Environment, Hebrew University, Jerusalem, Israel

N. Cordeiro $\cdot$ M. Faria

Competence Centre in Exact Science and Engineering,

University of Madeira, 9000-390 Funchal, Portugal

M. Mozetic

Department of Surface Engineering, Jozef Stefan Institute, Jamova cesta 39, 1000 Ljubljana, Slovenia 
2013; Wicklein and Salazar-Alvarez 2013). Nanocelluloses can be classified into two types: cellulose nanofibrils (CNFs) and cellulose nanocrystals (CNCs) which are different in morphology (Xu et al. 2013; Abdul Khalil et al. 2014). These novel forms of cellulose come from abundant and renewable natural sources, thus having low-cost, being economically beneficial in replacing synthetic fibers like carbon and glass. CNFs exhibit attractive properties such as the large specific surface area, very high elastic modulus, high aspect ratio, low thermal expansion, non-abrasive nature, non-toxic character and their ability to act as a significant reinforcement at low filler loading levels stimulates their use as reinforcing agents in polymer nanocomposites sector (Abdul Khalil et al. 2014; Jiang and Hsieh 2013b; Moon et al. 2011; Klemm et al. 2011; Saito et al. 2011). CNFs have great potential application in various fields, such as regenerative medicine, tissue engineering scaffolds, catalysis, textiles, surface coatings, drug delivery, food packaging and green nanocomposite materials (Klemm et al. 2011; Abdul Khalil et al. 2014; Sacui et al. 2014; Deng et al. 2010; Das et al. 2011). Besides these applications, CNFs have also been utilized to fabricate optically transparent films with excellent visible light transmittance and gas barrier properties (Fukuzumi et al. 2013; Saito et al. 2011; Moon et al. 2011).

Various types of plant fiber sources have been utilized for the extraction of nano-sized cellulose material. A lot of research work has been performed all over the world on the extraction of nanocellulose from a variety of native sources (Chirayil et al. 2014; Mathew et al. 2014; Sacui et al. 2014; Lin et al. 2014; Kumar et al. 2014; Fortunati et al. 2013; Silvério et al. 2013; Jiang et al. 2013a; Moran et al. 2008). CNFs can be obtained through mechanical treatments such as high pressure homogenization (Stenstad et al. 2008; Nakagaito and Yano 2004), high intensity ultrasonication (Chen et al. 2011), cryocrushing (Chakraborty et al. 2005), microfluidization (Ferrer et al. 2012), grinding (Panthapulakkal and Sain 2012) or/and chemical treatments such as typical hydrolysis (TEMPO-mediated oxidation, acid and enzymatic; Sacui et al. 2014). TEMPO-mediated oxidation is the most used method for the extraction of CNFs. This method allows the preparation of cellulose nanofibrils with high aspect ratios by oxidation of cellulose fibers via addition of $\mathrm{NaClO}$ in an aqueous solution in the presence of catalytic amounts of TEMPO and $\mathrm{NaBr}$ at
pH 10-11 (Isogai et al. 2011; Missoum et al. 2013). Abraham et al. (2011) reported a new approach for the extraction of cellulose nanofibrils through acid hydrolysis with oxalic acid at low concentrations which allows cellulose non degradation. Acid hydrolysis process breaks the disordered and amorphous parts of cellulose, releasing single and well-defined elementary cellulose nanofibrils (Jiang et al. 2013a). The nature of acid, acid concentration, the acid-to-cellulosic fibers ratio as well as temperature and time of reaction play an important role in the resulting morphology and properties of nanocellulose suspensions (Klemm et al. 2011; Eichhorn 2011; Habibi et al. 2010). It is known that the fundamental properties of native CNFs, such as morphology, crystallinity, aspect ratio and surface chemistry vary depending on the raw material source and the extraction process (Moon et al. 2011; Sacui et al. 2014). The variation is clearly illustrated in terms of length, microfibril angle, amount of non-cellulosic residues and quality. Therefore, the isolation of CNFs from different lignocellulosic biomass sources is relevant in order to efficiently compare and exploit these resources.

The present study report the characterization of cellulose nanofibrils isolated from five different sources (banana rachis, sisal, kapok, pineapple leaf and coir) using acid coupled steam explosion process. The main goal of the current experimental work is to examine the effect of the extraction processes and raw material sources on many of the key properties (structural morphology, crystallinity, surface energy, thermal stability and the yield) in the resulting nanocellulose. Isolated nanocellulose and their corresponding raw materials were analyzed and compared by a variety of characterization methods namely Fourier transform infrared (FTIR) spectroscopy, scanning electron microscopy (SEM), atomic force microscopy (AFM), transmission electron microscopy (TEM), birefringence, X-ray diffraction (XRD), inverse gas chromatography (IGC) and thermogravimetric analysis (TGA).

\section{Materials and methods}

\section{Materials}

Cellulose fibers were extracted from five different plant fiber sources (sisal, kapok, banana rachis, pineapple leaf and coir). The raw materials were 
collected from cultivation areas in Kollam, Alappuzha and Ernakulam districts in Kerala, India. All samples were air-dried and stored at room temperature $\left(32^{\circ} \mathrm{C}\right)$. The chemicals used in the extraction process were sodium hydroxide $(\mathrm{NaOH})$, sodium chlorite $\left(\mathrm{NaClO}_{2}\right)$, acetic acid $\left(\mathrm{CH}_{3} \mathrm{COOH}\right)$ and oxalic acid $\left(\mathrm{H}_{2} \mathrm{C}_{2} \mathrm{O}_{4}\right.$ $2 \mathrm{H}_{2} \mathrm{O}$ ). All the chemicals were reagent grade and used without further purification.

Extraction of nanocellulose

Nanocellulose were extracted using a combination of different chemical treatments such as alkaline treatment, bleaching and acid hydrolysis. The fibers obtained from various plant fibers were chopped into uniform size of approximately $10 \mathrm{~cm}$. Next, the fibers were subjected to steam explosion with $2 \% \mathrm{NaOH}$ (1:10 g/ml, fiber:liquor ratio) in an autoclave and kept under $20 \mathrm{lbs}$ pressure and at a temperature of 110 $120{ }^{\circ} \mathrm{C}$ for $1 \mathrm{~h}$. After releasing the pressure, the fibers were removed from the autoclave and subsequently washed in distilled water until the solution returned to neutral $\mathrm{pH}$. This purifying treatment eliminates noncellulosic components such as hemicelluloses, pectins and lignin from the fiber. The insoluble residue was further delignified with an acidified sodium chlorite solution $\left(1 \%, 15 \mathrm{ml} / \mathrm{g}\right.$ of fiber) at $70{ }^{\circ} \mathrm{C}$ for $1 \mathrm{~h}$. The process was repeated several times until the products became white. The cellulose pulp was neutralized with $\mathrm{NaOH}$ solution and thoroughly washed with distilled water. The resultant pulp was then dried in a vacuum oven at $60{ }^{\circ} \mathrm{C}$. Finally, the cellulose pulp was subjected to acid hydrolysis with $5 \%$ oxalic acid solution $(1: 15 \mathrm{~g} /$ $\mathrm{ml}$, cellulose pulp:oxalic acid) for $3 \mathrm{~h}$ in an autoclave until it attained a pressure of $20 \mathrm{lbs}$, then the pressure was released immediately. The fibers were taken out, filtered and rinsed with distilled water until $\mathrm{pH}$ reached seven. After these treatments, the fibers were suspended in water and then subjected to mechanical stirring for $6 \mathrm{~h}$. The resulting aqueous suspensions were preserved for further characterizations. The yields of nanocellulose isolated from various sources were calculated gravimetrically using the Eq. 1;

Nanocellulose yield $(\%)$

$$
\begin{aligned}
= & \frac{\text { Weight of dried nanocellulose }(\mathrm{g})}{\text { Weight of raw fiber used for extraction }(\mathrm{g})} \\
& \times 100
\end{aligned}
$$

The percentage yield of nanocellulose is different among different sources and the values are given in Table 1.

\section{Characterization methods}

\section{Fourier transform infrared spectroscopy (FTIR)}

FTIR spectra of the untreated plant fibers, bleached fibers and nanocellulose obtained from various sources were recorded on a Fourier transform infrared (FTIR) instrument (Perkin-Elmer PC1600, USA) using ATR apparatus, in the range of $4,000-400 \mathrm{~cm}^{-1}$ with a spectral resolution of $4 \mathrm{~cm}^{-1}$. The samples were dried in an air oven at $60{ }^{\circ} \mathrm{C}$ for $8 \mathrm{~h}$ before carrying out the analysis. The FTIR spectra of the samples were taken directly and not in the form of ultrathin pellets by $\mathrm{KBr}$ disk method.

\section{Scanning electron microscopy (SEM)}

The surface morphology of cellulosic fibers was observed using a scanning electron microscope model JEOL JSM-6390 with an accelerating voltage of 10-20 kV. Prior to SEM examination, the samples were oven dried at $60{ }^{\circ} \mathrm{C}$ for $8 \mathrm{~h}$ and then a fine layer of gold was deposited on samples by means of a plasma sputtering apparatus.

\section{Atomic force microscopy (AFM)}

Surface topography and morphology of the samples were analysed using a NT-MDTs Solver PRO atomic force microscope (AFM). Silicon cantilevers with a typical resonant frequency of $240 \mathrm{kHz}$ and spring constant of $11.8 \mathrm{~N} / \mathrm{m}$ were used to acquire images in tapping mode at room temperature under ambient conditions. The scanning rate was around $1.5 \mathrm{~Hz}$.

Table 1 Percentage yield of nanocellulose isolated from various sources

\begin{tabular}{ll}
\hline Nanocellulose source & Yield (\%) \\
\hline Banana rachis & 28.6 \\
Sisal & 38.8 \\
Kapok & 33.7 \\
Pineapple leaf & 40.1 \\
Coir & 23.5 \\
\hline
\end{tabular}


Transmission electron microscopy (TEM)

The size of the elementary particles was determined by TEM. Prior to deposition on the grid, the nanocellulose suspensions were sonicated (60 s) with ice bath for three times and diluted to $100 \mu \mathrm{g} / \mathrm{ml}$. Drops $(5 \mu \mathrm{l}$ each) were deposited on glow-discharged carboncoated copper TEM grids for $1 \mathrm{~min}$ and blotted. No staining was used. The grids were observed with a Tecnai-12 (Philips) transmission electron microscope operated at $120 \mathrm{kV}$. The specimens were observed under low-dose conditions. Images were captured digitally on a TVIPSF224 CCD camera.

\section{Birefringence analysis}

Samples for birefringence analysis were prepared by ultrasonicating the aqueous suspension of nanocellulose in an ice bath for $20 \mathrm{~min}$. Birefringence properties of nanocellulose suspensions ( $3 \% \mathrm{w} / \mathrm{w})$ were detected between crossed polars in a dark box. The ordering present in aqueous suspensions of nanocellulose was also studied in the solid phase by film casting the suspension at ambient conditions. Images were captured with a Nikon DS Camera control unit DS-U2 on a Nikon Polarizing Microscope Eclipse LV100POL.

\section{$X$-ray diffraction $(X R D)$ analysis}

X-ray diffraction analysis of nanocellulose from various sources were conducted using an X-ray diffractometer (Bruker AXS D8 Advance, Germany), equipped with $\mathrm{Cu} \mathrm{K} \alpha$ radiation source (wavelength $=0.154 \mathrm{~nm}$ ) operating at $40 \mathrm{kV}$ and $30 \mathrm{~mA}$. The XRD patterns were recorded over the angular range $2 \theta=5-40^{\circ}$.

The crystallinity index (Ic) was calculated from the heights of the 200 peak $\left(\mathrm{I}_{200}, 2 \theta=22.6^{\circ}\right)$ and the intensity minimum between the 200 and 110 peaks $\left(\mathrm{I}_{\mathrm{am}}, 2 \theta=18^{\circ}\right)$ using the Segal method (Segal et al. 1959) as shown in Eq. 2. $I_{200}$ represents both crystalline and amorphous material, whereas $\mathrm{I}_{\mathrm{am}}$ represents the amorphous material. Crystal size of the nanocellulose is calculated using Scherrer formula as shown in Eq. 3.

Crystallinity index $(\%)=\frac{I_{200}-I_{a m}}{I_{200}} \times 100$
Crystal size $(\mathrm{D})=0.89 \lambda / \beta \operatorname{Cos} \theta$

where $\lambda$ is the $\mathrm{X}$-ray wavelength, $\beta$ is the angular width at half maximum intensity and $\theta$ is the Bragg angle.

Inverse gas chromatography (IGC) analysis

Surface properties were analyzed using a commercial inverse gas chromatography (Surface Measurements Systems, London, UK). The measurements and theory applied were described exhaustively by Cordeiro et al. (2011). In this study, all the measurements were carried out at $0 \%$ relative humidity $(\mathrm{RH})$, a helium flow rate of $10 \mathrm{ml} / \mathrm{min}$ and in duplicate producing an experimental variation below $4 \%$. Methane was used as reference molecule. Heptane, octane, nonane and decane were the four $n$-alkanes used for determination of the dispersive component of the surface free energy at three temperatures: 294, 298 and 302 K. Acetonitrile $(\mathrm{ACN})$, ethyl acetate (EtOAc), ethanol (EtOH), acetone (AC) and tetrahydrofuran (THF) were used for determination of the Gibbs specific free energy $\left(\Delta G_{S}^{S P}\right)$ and acid-base surface character $\left(K_{A}\right.$ and $\left.K_{B}\right)$ at $298 \mathrm{~K}$. $n$-octane was used to measure the parameters of isotherm (surface area, $S_{B E T}$, and monolayer capacity, $n_{m}$ ) and permeability (Adsorption potential, $A_{\max }$ ) at $298 \mathrm{~K}$.

\section{Thermogravimetric analysis (TGA)}

Thermal degradation characteristics of the five natural fiber sources were carried out using a thermogravimetric analyzer (Perkin-Elmer STA 6000). The thermal analyses were conducted in a nitrogen atmosphere (flow rate of $100 \mathrm{ml} / \mathrm{min}$ ) with a constant heating rate of $10{ }^{\circ} \mathrm{C} / \mathrm{min}$ and at a temperature of $25-700{ }^{\circ} \mathrm{C}$. The weight loss $(\%)$ at the cellulose decomposition temperature was determined, as all as the residual char (\%) at $700{ }^{\circ} \mathrm{C}$.

\section{Results and discussion}

Fourier transform infrared spectroscopy (FTIR) analysis

Cellulose fibers obtained from five different sources were analyzed using FTIR spectroscopy to observe the 
changes in chemical composition of fibers before and after the chemical treatments. Figure 1 shows the FTIR spectra of the untreated plant fibers, bleached cellulose fibers and nanocellulose fibers obtained from banana rachis, sisal, kapok, pineapple leaf and coir respectively. The peak at $1,732 \mathrm{~cm}^{-1}$ in untreated plant fiber is attributed to $\mathrm{C}=\mathrm{O}$ stretching of the acetyl and uronic ester groups of hemicellulose or the ester linkage of carboxylic groups of ferulic and $p$-coumaric acids of lignin (Alemdar and Sain 2008). The peak at $1,507 \mathrm{~cm}^{-1}$ in the spectrum of untreated plant fibers is attributed to the $\mathrm{C}=\mathrm{C}$ stretching vibrations of the aromatic rings of lignin (Sain and Panthapulakkal
2006). These peaks are reduced in the FTIR spectra of bleached cellulose fibers and acid hydrolysed nanocellulose indicating the removal of hemicellulose and lignin from the fibers during the extraction process. The peaks observed at 2,916 and $1,629 \mathrm{~cm}^{-1}$ are associated with the stretching vibrations of $\mathrm{C}-\mathrm{H}$ and $\mathrm{O}-\mathrm{H}$ groups respectively. The spectral bands observed in the region around $1,647-1,638 \mathrm{~cm}^{-1}$ are attributed to the $\mathrm{O}-\mathrm{H}$ bending of the adsorbed water (Mandal and Chakrabarty 2011). The bands at 1,151 and $1,105 \mathrm{~cm}^{-1}$ ascribed to the $\mathrm{C}-\mathrm{C}$ ring stretching and $\mathrm{C}-\mathrm{O}-\mathrm{C}$ glycosidic ether linkages respectively (Mandal and Chakrabarty 2011).
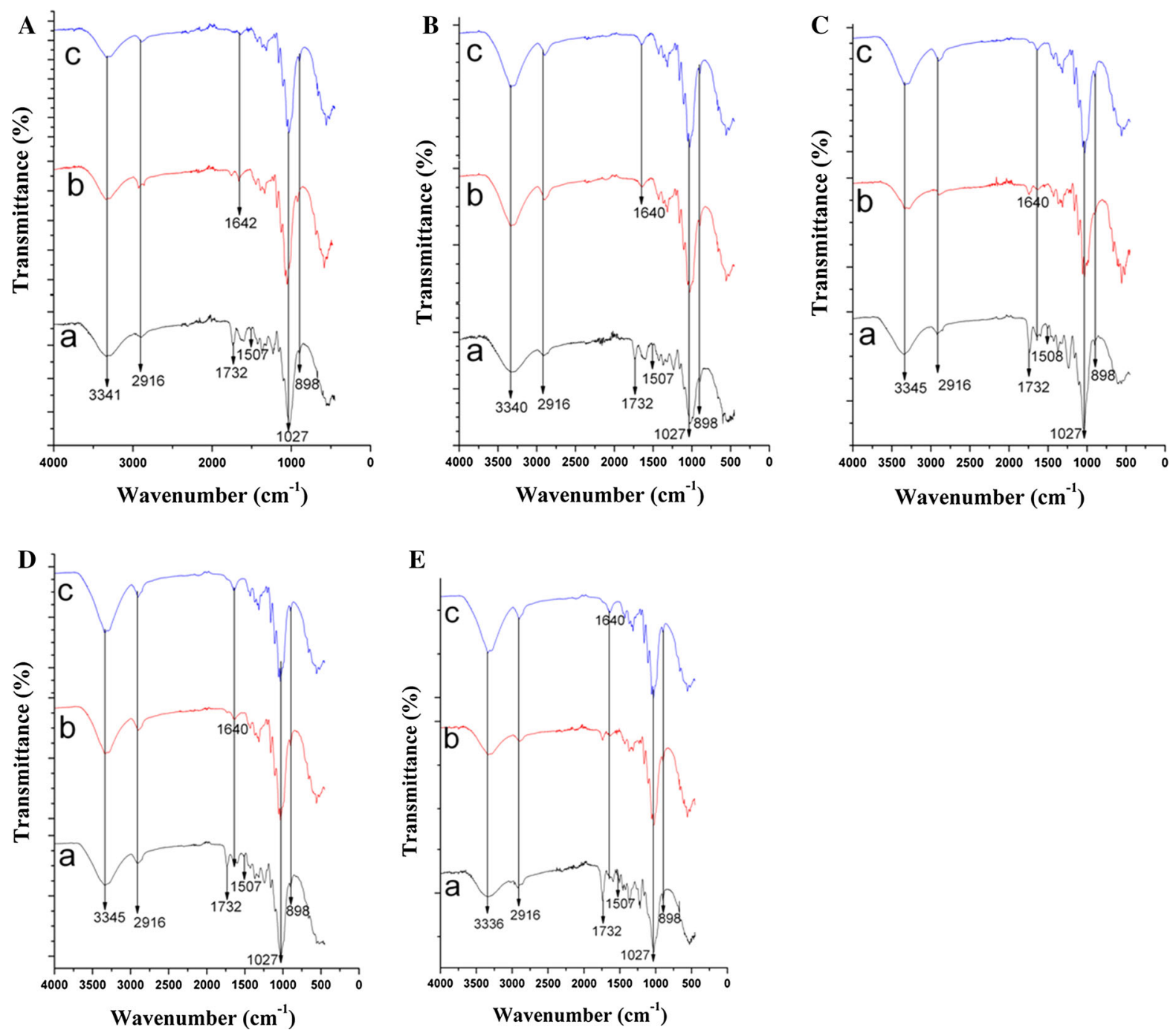

Fig. 1 FTIR spectra of $a$ untreated plant fibers $b$ bleached cellulose fibers and $c$ nanocellulose of A banana rachis B sisal C kapok D pineapple leaf and $\mathbf{E}$ coir 
The most significant absorption bands observed in the FTIR spectra of all the samples (untreated plant fiber, bleached cellulose fibers and acid hydrolysed nanocellulose) at 1,027 and $898 \mathrm{~cm}^{-1}$ are assigned to the $\mathrm{C}-\mathrm{O}-\mathrm{C}$ stretching vibration of pyranose ring and the glycosidic linkages between glucose units in cellulose, respectively (Alemdar and Sain 2008). All the samples also exhibited a broad absorption band in the region $3,500-3,300 \mathrm{~cm}^{-1}$ corresponding to the free $\mathrm{O}-\mathrm{H}$ stretching vibration of the $\mathrm{OH}$ groups in cellulose molecules (Chirayil et al. 2014). FTIR spectrum of nanocellulose extracted from all the five sources is identical, and there are no significant differences among the sources.

Scanning electron microscopic (SEM) analysis

Figure 2 shows the comparison of the SEM images of raw cellulose fibers obtained from the five sources. The raw fiber surface appears to be smooth due to the presence of an outer non-cellulosic layer composed of cementing materials including lignin, hemicelluloses, pectin, wax and oil. Figure 3 shows the comparison of the SEM images of bleached cellulose fibers extracted from the five sources. In this stage, fiber bundles were separated into individual micro-sized fibers, which can be clearly seen in Fig. 3. The removal of hemicelluloses and lignin with some other extractives during the chemical treatments was the main reason for such observation, as evident from the FTIR analysis.
Figure 4 shows the comparison of the SEM images of the oven dried acid hydrolyzed cellulose fibers. Upon drying, all the five nanocellulose samples tend to self-assembled into sub-micron wide and micrometer long fibers. This may be due to the increase in strong inter-fibrillar attraction via hydrogen bonding among the surface hydroxyl groups of cellulose during the drying process (Jiang and Hsieh 2013b). The reduction in fiber diameter is observed in all the fiber samples after acid hydrolysis due to the successful removal of amorphous phase from the fiber samples. This fact was confirmed by AFM and TEM analyses. The fibers were well-individualized to nano-dimension after acid hydrolysis followed by mechanical agitation and can be observed clearly from the AFM and TEM images (Figs. 5, 6). Nanocellulose with rectangular and rod shaped structures were obtained due to effective acid hydrolysis.

Atomic force microscopic (AFM)

and transmission electron microscopic (TEM) analyses

Morphological examination of the nanocellulose is essential because the source of the cellulose and the hydrolysis technique has a large impact on the dimension and properties of nanocellulose. Thus, AFM and TEM analyses were performed to investigate the morphology and size of the nanocellulose samples obtained from different sources. Figure 5

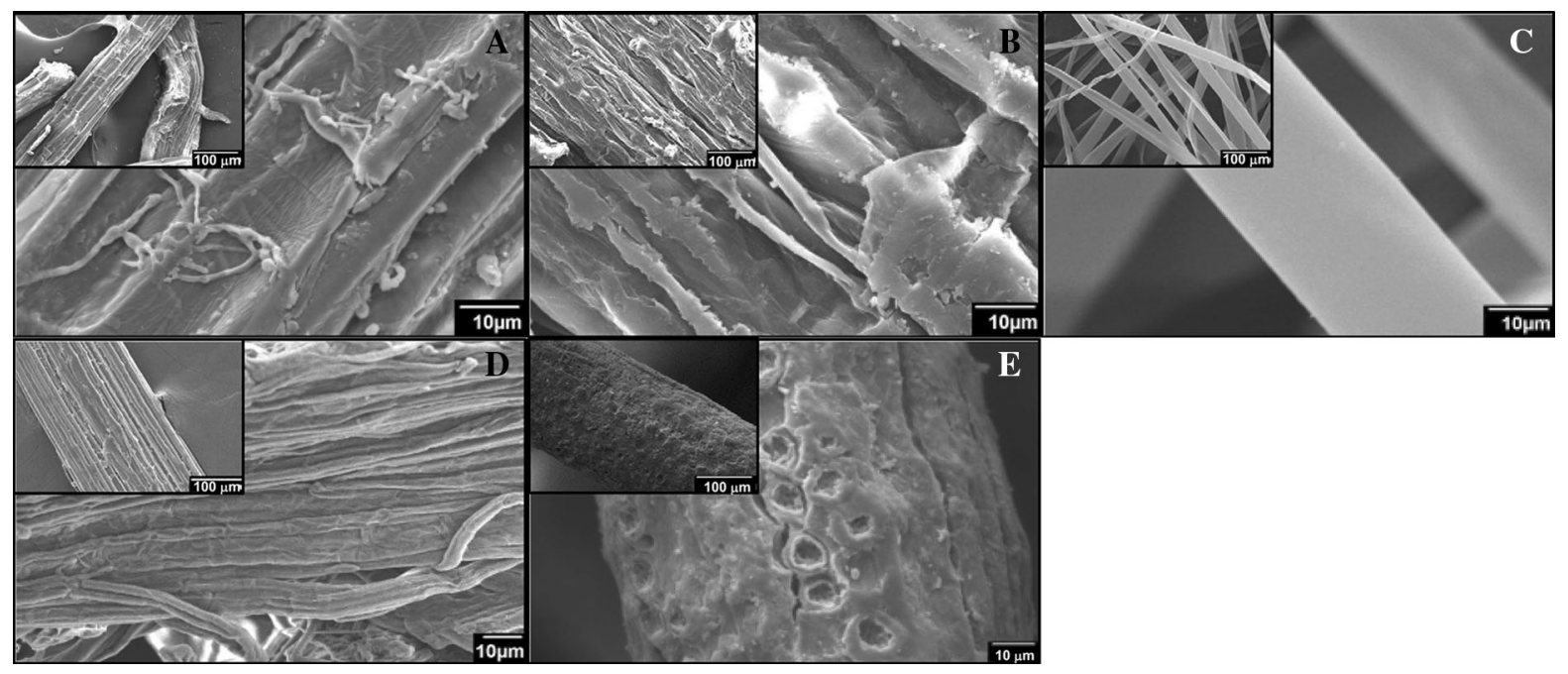

Fig. 2 SEM images of untreated plant fibers obtained from a banana rachis $\mathbf{b}$ sisal $\mathbf{c}$ kapok $\mathbf{d}$ pineapple leaf and $\mathbf{e}$ coir 


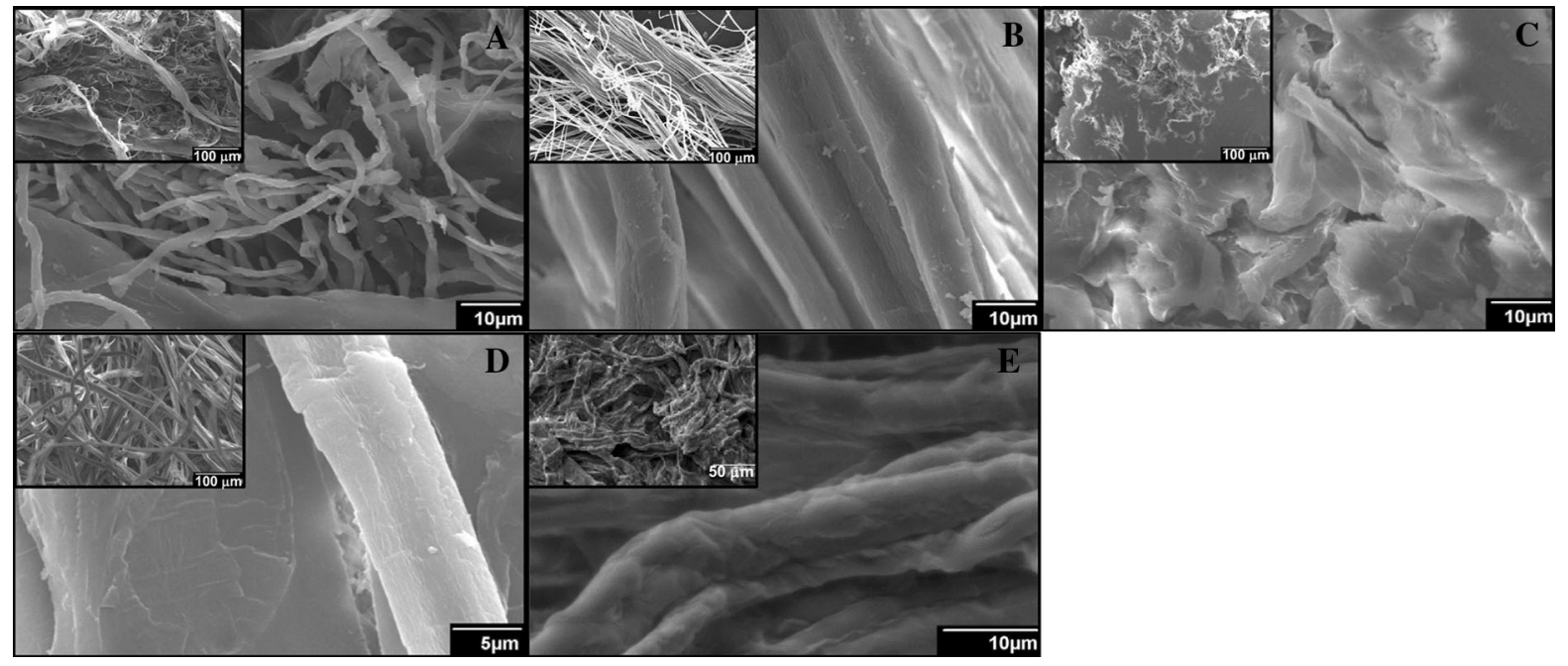

Fig. 3 SEM images of bleached cellulose fibers obtained from $\mathbf{a}$ banana rachis $\mathbf{b}$ sisal $\mathbf{c}$ kapok $\mathbf{d}$ pineapple leaf and e coir

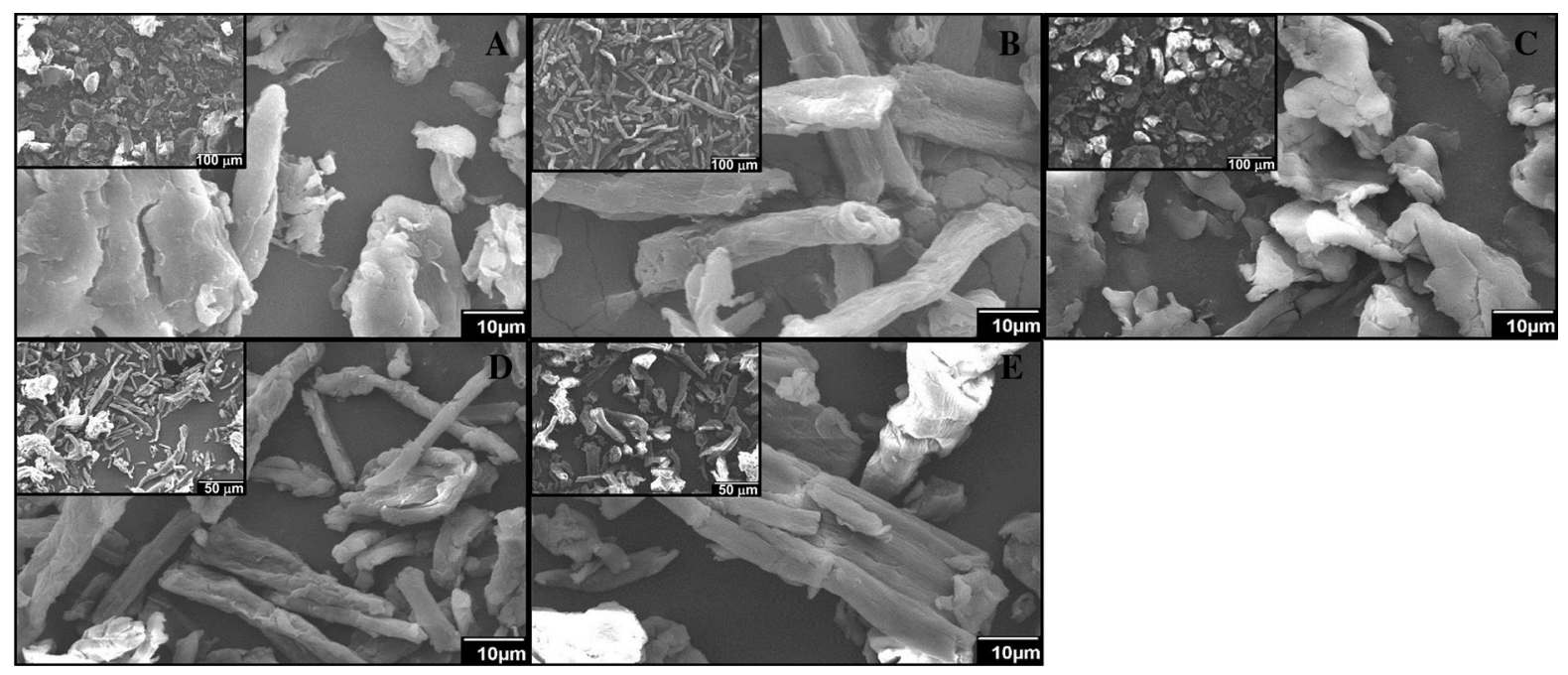

Fig. 4 SEM images of acid hydrolyzed cellulose fibers obtained from a banana rachis $\mathbf{b}$ sisal $\mathbf{c}$ kapok $\mathbf{d}$ pineapple leaf and $\mathbf{e}$ coir

shows the AFM topography images of the isolated nanocellulose of banana rachis, sisal, kapok, pineapple leaf and coir respectively. The images shown on the right side of Fig. 5 correspond to cross-section profile marked with green lines on the left side of the image. The length of this line is then enlarged to get some curves on the right side of the image. This provides the outline of appointed structure. The numbers on the bottom and the left side of the morphology profile corresponds to the cross-section length and height of the defined structure. The diameter of CNF is deduced from the cross-section length.
AFM images of nanocellulose revealed the effective isolation of nanocellulose from the microsized fibers. Nanocellulose isolated from different sources appeared to be aggregated rod-like nanofibrilar network-structures. Aggregation and overlapping of individual cellulose nanofibrils make difficult the accurate determination of size and aspect ratio of the nanocellulose samples using AFM technique. For this reason, AFM analysis was conducted on a small part of the surface of individual nanocellulose particles. The diameters of the nanocellulose structures varied considerably among the sources. The approximate 


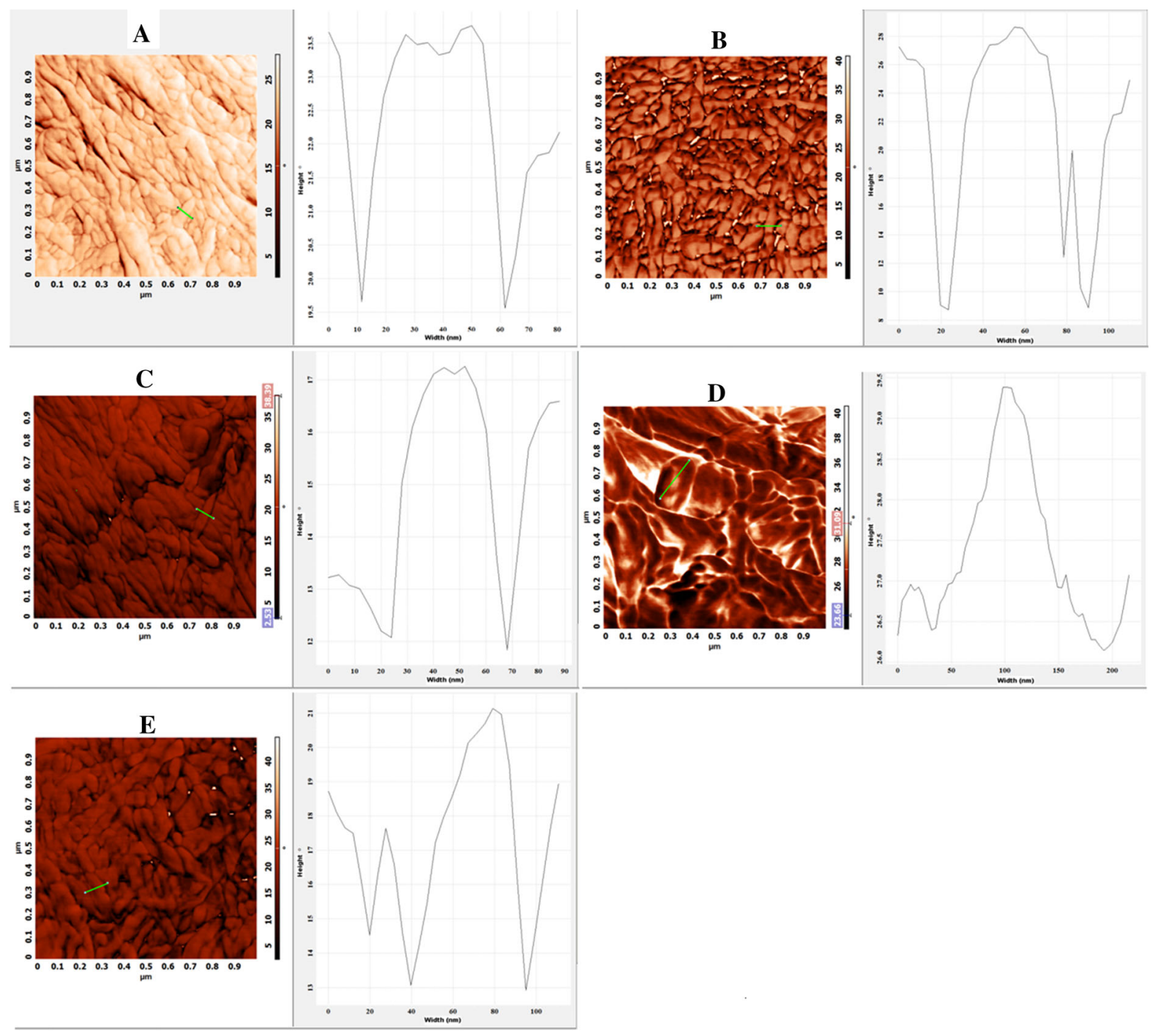

Fig. 5 AFM images of nanocellulose obtained from a banana rachis $\mathbf{b}$ sisal $\mathbf{c}$ kapok $\mathbf{d}$ pineapple leaf and e coir

diameters for most of the nanocellulose fibers obtained from banana rachis, sisal, kapok, pineapple leaf and coir were found to be between 10-60, 20-80, 20-70, 50-150 and 40-90 $\mathrm{nm}$ respectively. The calculated width of the nanocellulose particles is larger than the actual value due to the severe tip broadening effect in AFM measurements. The tip broadening effect is less pronounced in the rod-shaped cellulose nanofibrils compared to the spherical shaped nanoparticles. Therefore, the calculated width of the nanocellulose samples mentioned above may be approximately $10 \%$ more than the actual value.

Transmission electron microscopy images were taken to examine the clear difference between the width and the structure of the nanocellulose samples. TEM images (Fig. 6) strongly support the isolation of individualized cellulose nanofibrils from various sources. The morphology and dimension of the cellulose nanofibrils varied among different sources. For example, cellulose nanofibrils isolated from sisal, pineapple leaf and coir appear to be long, flexible and entangled, however banana rachis and kapok appear to be much more individualized and rod-like structures. The size measurements from TEM images showed that there would be some variation in fiber diameter for different sources. The average width of cellulose nanofibrils of banana, sisal, kapok, pineapple leaf and coir were found to be $18 \pm 7,13 \pm 4,16 \pm 6,22 \pm 3$ 

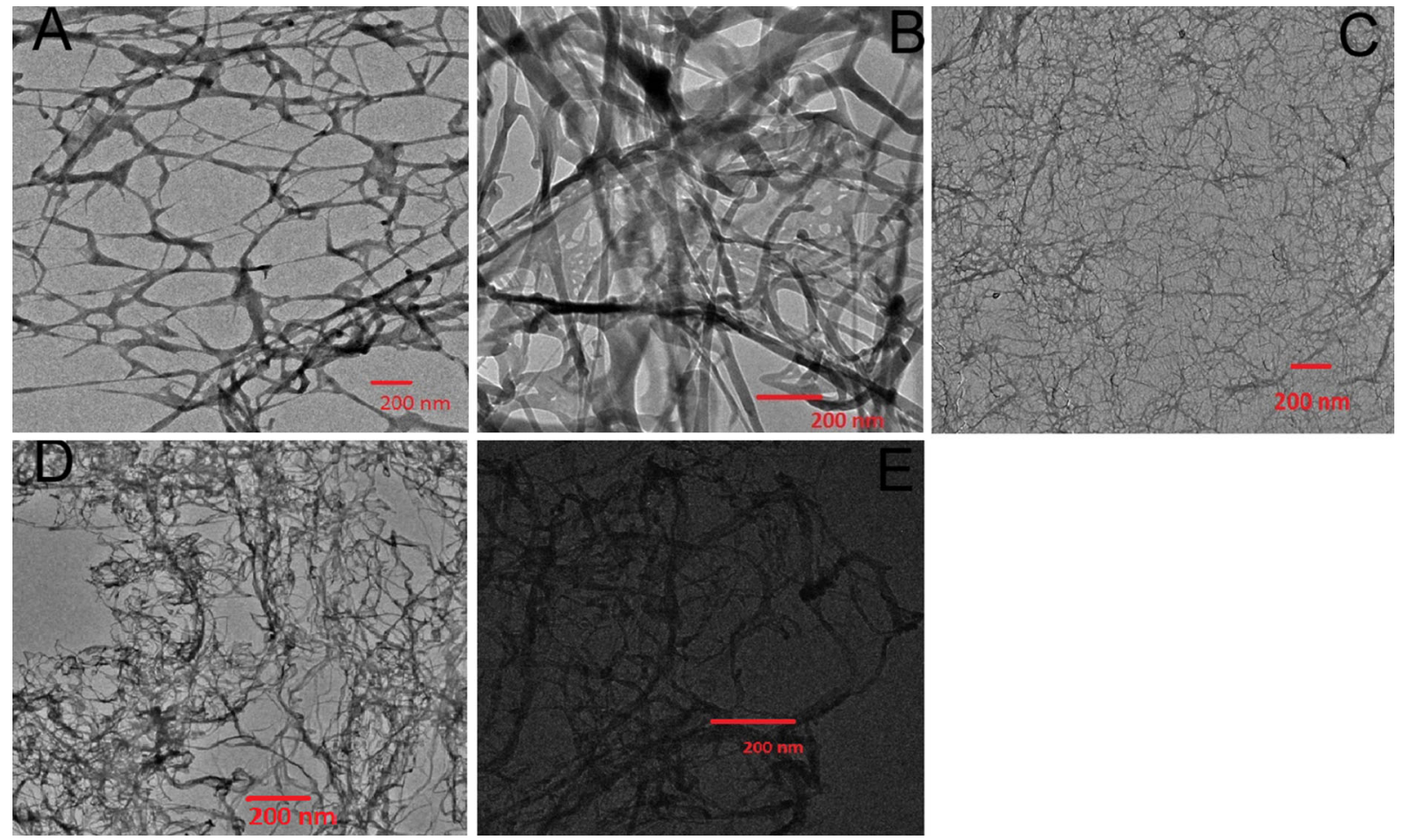

Fig. 6 TEM images of nanocellulose obtained from a banana rachis $\mathbf{b}$ sisal $\mathbf{c}$ kapok $\mathbf{d}$ pineapple leaf and e coir

and $14 \pm 5 \mathrm{~nm}$ respectively. Similar result for cellulose nanofibrils obtained by different extraction methods were reported by Sacui et al. (2014), Xu et al. (2013), Wang et al. (2012), Abraham et al. 2011 and Kaushik and Singh (2011).

The exact length of the cellulose nanofibrils cannot be measured accurately because these fibrils appear to be bundled and overlapped with each other, may be due to the formation of interfibrillar hydrogen bonds. From the TEM images, can be predict that these cellulose nanofibrils have several micrometers in length and therefore the aspect ratio (length to diameter ratio) of the isolated nanofibrils is expected to be very high. The aspect ratio of the fibrils is an important material parameter in the processing of composites with good mechanical properties. The higher the aspect ratio of the fibrils, the better the composite mechanical properties, even at low concentrations (Moon et al. 2011).

\section{Birefringence analysis}

Birefringence analysis was used to confirm the presence of isolated nanocellulose crystals in aqueous suspension and is considered to be an effective method to prove good dispersibility in suspensions (Silvério et al. 2013). Birefringence in nanocrystalline cellulose is known to result either from a structural anisotropy form of cellulose or the flow anisotropy due to the alignment of the nanocrystals underflow (Silvério et al. 2013). The flow birefringence properties of aqueous suspensions of nanocellulose $(3 \% \mathrm{w} / \mathrm{w})$ obtained after ultrasonication process were investigated. Since the nanocelluloses are in fibrils form which is clear from its TEM analyses, ultrasonication will break the amorphous regions of the fibers leading to the accumulation of cellulose nanocrystals to get birefringence results. Figure 7a shows the photograph of the aqueous suspension of nanocellulose extracted from banana rachis fibers. Figure $7 \mathrm{~b}$ shows the flow birefringence character of nanocellulose suspensions observed between crossed polars in shearing conditions. The aqueous suspensions of nanocellulose exhibited strong shear-induced birefringence, highlighting the ability of the nanocellulose under study to form a chiral nematic liquid crystalline phase in equilibrium with isotropic phase (Fortunati et al. 2013). The self assembly of chiral nematic phases by 

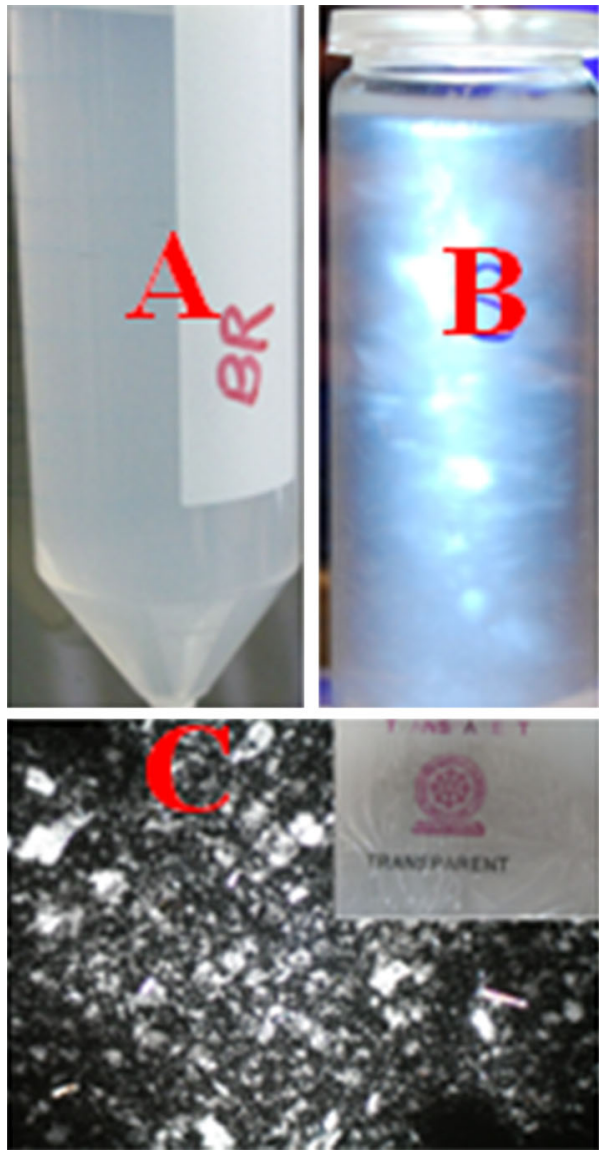

Fig. 7 a Photograph of aqueous suspension of nanocellulose b birefringence of nanocellulose observed between crossedpolars and $\mathbf{c}$ birefringence of transparent nanocellulose films made by evaporation of water

suspensions of nanocellulose has received an increased amount of attention for its potential applications. The ordering observed in aqueous suspensions of nanocellulose with $3 \mathrm{wt} \%$ concentration was also studied in the solid phase after the evaporation of water (Fig. 7c). The transparent films obtained by simply casting the suspensions of nanocellulose exhibit a smooth surface texture. Nanocellulose isolated from other four sources also show similarity in birefringence properties.

$\mathrm{X}$-ray diffraction analysis

XRD studies of untreated plant fibers and treated cellulose fibers were performed to investigate the crystalline behavior of these fibers. Figure 8 shows the comparison of XRD patterns of nanocellulose isolated

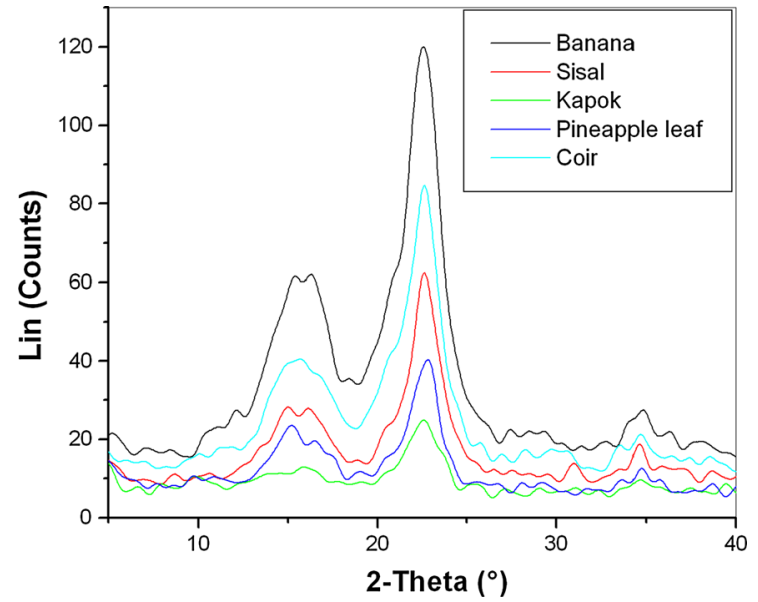

Fig. 8 XRD patterns of nanocellulose isolated from the five different biomass sources

Table 2 XRD analysis parameters for crystallinity index (\%) and crystal size $(\mathrm{nm})$ for the nanocellulose isolated from various sources

\begin{tabular}{lll}
\hline Sample & Crystallinity index $(\%)$ & Crystal size $(\mathrm{nm})$ \\
\hline Banana rachis & 80.9 & 3.4 \\
Sisal & 91.3 & 3.7 \\
Kapok & 86.5 & 3.5 \\
Pineapple leaf & 92.3 & 3.7 \\
Coir & 84.5 & 3.4 \\
\hline
\end{tabular}

from the five different sources. All the diffractograms showed two peaks around $2 \theta=16.5^{\circ}$ and $22.6^{\circ}$, which are supposed to represent the typical cellulose I structure (Nishiyama et al. 2003). The peak at about $2 \theta=16^{\circ}$ was found to be a combination of two peaks, which indicate the presence of some small amount of cellulose II structure. The higher crystalline nature of nanocellulose is undoubtedly due to the more efficient removal of non-cellulosic components from the fibers, i.e. the dissolution of amorphous regions, by controlled acid hydrolysis process.

The values of $\%$ crystallinity and the crystal size for nanocellulose obtained from the five sources are listed in Table 2. The crystallinity index of sisal, kapok and coir were found to be around 91.3, 86.5 and $84.5 \%$ respectively. The highest and the lowest value of crystallinity were obtained for pineapple leaf $(92.3 \%)$ and banana rachis $(80.9 \%)$ nanocellulose respectively. The crystal size of the nanocellulose extracted 
from all the five sources were found to be around $3.5 \mathrm{~nm}$. This value of crystal size was in the same range as reported for nanocellulose isolated from sugarcane bagasse (Kumar et al. 2014). The results of XRD analysis clearly confirmed that the degree of crystallinity of the nanocellulose depend on the cellulosic raw material.

Inverse gas chromatography (IGC)

IGC analysis was used to observe the effects of the chemical treatment on the surface properties of the five lignocellulosic renewable materials under study. The knowledge of the nanocellulose surface properties has a great interest to predict its compatibility with other molecules/polymers. The surface proprieties of cellulosic materials are known to depend on the chemical composition of the material (the content of the aliphatic components, cellulose, hemicelluloses and lignin), crystallinity and the arrangement/orientation of chemical groups in the surface (Cordeiro et al. 2012). Table 3 resume the results obtained in the

Table 3 IGC results (298 K) of untreated plant fibers, bleached cellulose fibers and the isolated nanocellulose from banana rachis, sisal, kapok, pineapple leaf and coir

\begin{tabular}{llll}
\hline Sample & $\gamma_{S}^{D}\left(\mathrm{~mJ} / \mathrm{m}^{2}\right)$ & $K_{B} / K_{A}$ & $S_{B E T}\left(\mathrm{~m}^{2} / \mathrm{g}\right)$ \\
\hline RB & 43.98 & 2.49 & 0.68 \\
BB & 42.94 & 0.44 & 1.05 \\
BNC & 43.77 & 1.07 & 0.86 \\
RS & 35.86 & 1.88 & 0.66 \\
BS & 39.10 & 0.22 & 1.73 \\
SNC & 40.69 & 1.17 & 0.82 \\
RK & 32.38 & 1.00 & 1.10 \\
BK & 42.10 & 0.30 & 1.12 \\
KNC & 49.49 & 1.00 & 0.88 \\
RP & 41.24 & 2.22 & 0.85 \\
BP & 42.59 & 0.56 & 1.14 \\
PNC & 40.71 & 1.60 & 0.82 \\
RC & 47.72 & 2.36 & 0.83 \\
BC & 43.24 & 0.57 & 1.98 \\
CNC & 48.37 & 1.83 & 0.99 \\
\hline
\end{tabular}

$R B$ raw banana rachis, BB bleached banana rachis, $B N C$ banana rachis nanocellulose, $R S$ raw sisal, $B S$ bleached sisal, $S N C$ sisal nanocellulose, $R K$ raw kapok, $B K$ bleached kapok, $K N C$ kapok nanocellulose, $R P$ raw pineapple leaf, $B P$ bleached pineapple leaf, $P N C$ pineapple leaf nanocellulose, $R C$ raw coir, $B C$ bleached coir, $C N C$ coir nanocellulose surface characterization of the raw material, bleached cellulose fibers and the acid hydrolyzed samples. The fiber treatments did not change greatly the dispersive surface energy $\left(\gamma_{S}^{D}\right)$ of the banana, pineapple and coir samples. A significant variation was found in the kapok and sisal (53 and $13 \%$ respectively). As seen in Fig. 9, the chemical treatment decrease the number of the active sites in the fibers surface but increase their energy. However, the fibers after the bleaching treatment present similar energy as the raw material but with the highest amount of actives site, due to the higher surface area $\left(S_{B E T}\right.$; Table 3$)$.

The nanocellulose obtained from the five materials show a dispersive component of the surface energy between 40.69 ( sisal) and $49.49 \mathrm{~mJ} / \mathrm{m}^{2}$ (kapok), which make the nanocellulose from kapok the more hydrophobic nanocellulose surface.

The interaction between the polar probes and the samples indicate the presence of both acidic and basic active sites on the samples surface. The values of $\mathrm{K}_{\mathrm{a}}$ and $\mathrm{K}_{\mathrm{b}}$ for the raw materials show a Lewis
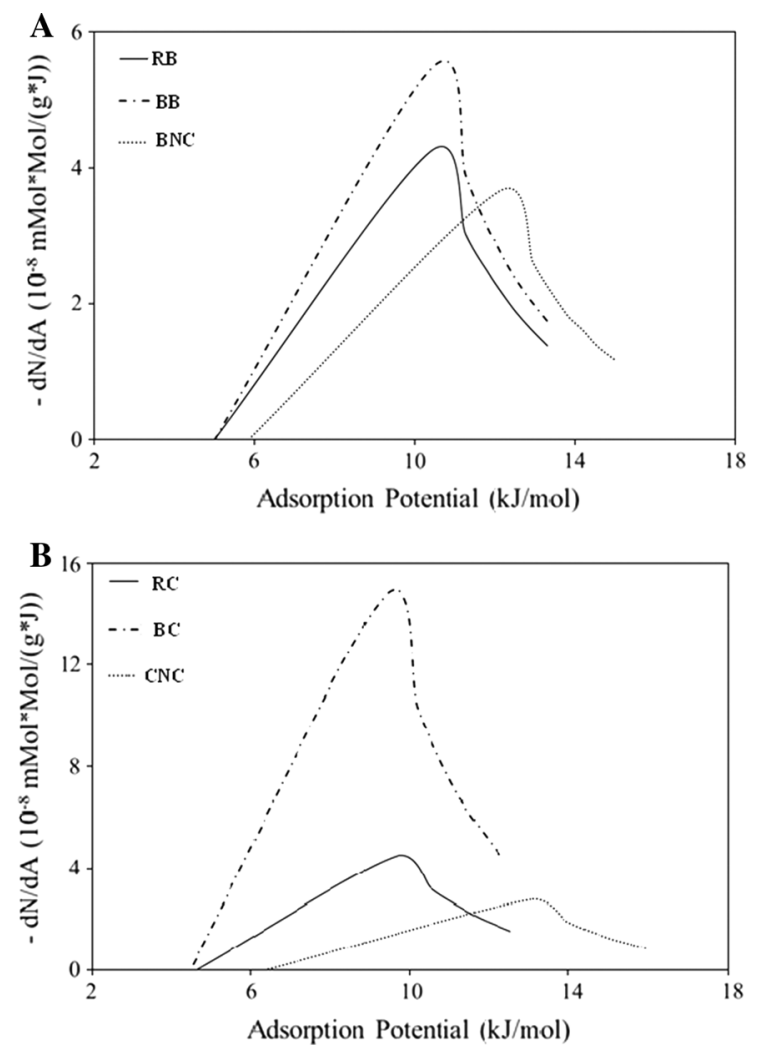

Fig. 9 Heterogeneity profiles obtained with $n$-octane onto $\mathbf{a}$ banana and $\mathbf{b}$ coir samples 
basic character, with a $\mathrm{K}_{\mathrm{b}} / \mathrm{K}_{\mathrm{a}}$ ratio between 1.00 and 2.49 , corresponding to kapok and banana rachis, respectively.

After the bleaching treatment, the surface had a strong acidification $\left(\mathrm{K}_{\mathrm{b}} / \mathrm{K}_{\mathrm{a}}\right.$ between 0.22 and 0.57$)$, due to the decrease of the basic sites. A more basic character at the surface was recovered after acid hydrolyze and makes the nanocellulose from coir, pineapple leaf and sisal with a predominantly basic surface, suggesting better potential in applications with acid agents. Banana rachis and kapok nanocellulose present an amphoteric surface character which allows specific intermolecular interactions with acid and basic solvents, plasticizers, polymers or fillers, important for practical applications.

Based on the obtained linear adsorption isotherms the surface area $\left(S_{B E T}\right)$ values were determined (Table 3 ). The $S_{B E T}$ of the raw fibers did not change greatly and has ranged between 0.66 and $0.85 \mathrm{~m}^{2} / \mathrm{g}$. Due to the nature of kapok, that present a more fibrous material (see SEM Fig. 2c, 3c), the $S_{B E T}$ has a higher value $\left(1.10 \mathrm{~m}^{2} / \mathrm{g}\right)$. With this exception (kapok), the IGC data shows that the bleached fibers have a surface area about two times higher than the raw fibers, due to the defibrillation induced by the chemical processes. However, a decrease in $S_{B E T}$ was obtained to the nanocellulose, eventhough there is a decrease in the particle size. The surface area reflects not only the size of the particle but also the shape, porosity and surface roughness of the sample. The nanocellulose present high crystallinity, compact rectangular and rod shaped structures with reduced diameter, which explain the $S_{B E T}$ decrease when compared to the bleached fibers with higher porosity at the surface. Similar $S_{B E T}$ values $\left(0.82-0.99 \mathrm{~m}^{2} / \mathrm{g}\right)$ were obtained for the nanocellulose from the five lignocellulosic renewable materials.

\section{Thermogravimetric analysis (TGA)}

The determination of thermal stability of nanocellulose is a key factor for its potential use as reinforcing agent in bionanocomposites. The thermal stability of polymeric material is known to depend on the sample inherent characteristics as well as on the molecular interactions between the different macromolecules (Maiti et al. 2013). Thermal stability of untreated plant fibers and extracted nanocellulose were investigated by the thermogravimetric method. Figure 10 shows the TGA and differential thermogravimetric (DTG) curves of the untreated plant fibers, bleached cellulose fibers and the acid hydrolyzed specimen of nanocellulose from banana rachis, sisal, kapok, pineapple leaf and coir respectively. The details of major decomposition temperatures and the corresponding percentage of weight losses are summarized in Table 4.

All the TGA curves show an initial small weight loss between 50 and $100{ }^{\circ} \mathrm{C}$ corresponding to the evaporation of moisture from these materials. The thermal degradation of untreated plant fibers shows several stages, indicating the presence of different components that decompose at different temperatures. The first degradation corresponding to the evaporation of chemisorbed water is found at a temperature close to $70{ }^{\circ} \mathrm{C}$ (weight loss $2.8 \%$ ) for banana rachis, at $80{ }^{\circ} \mathrm{C}$ (weight loss $3.3 \%$ ) for sisal, at $56{ }^{\circ} \mathrm{C}$ (weight loss $0.78 \%$ ) for kapok, at $59{ }^{\circ} \mathrm{C}$ (weight loss $1.8 \%$ ) for pineapple leaf and at $82{ }^{\circ} \mathrm{C}$ (weight loss $2.1 \%$ ) for coir. The second degradation occurs at a temperature ranging from 220 to $310{ }^{\circ} \mathrm{C}$, resulting mainly from the thermal decomposition of hemicelluloses and some portion of lignin. This peak is reduced after bleaching treatment and disappeared, as expected, in the case of extracted nanocellulose. The final major decomposition peak observed at the high temperature range (300-400 ${ }^{\circ} \mathrm{C}$ ) is accounted for the pyrolysis of cellulose. Almost all the cellulose component was decomposed at this temperature. However, the presence of phenyl groups in lignin made it difficult to decompose since its decomposition extended to the whole temperature range, starting below $200{ }^{\circ} \mathrm{C}$ and up to $700{ }^{\circ} \mathrm{C}$. In the case of untreated plant fibers, the peak temperatures corresponding to the degradation of cellulose was found to be at around 330, 331, 346, 337 and $353{ }^{\circ} \mathrm{C}$ for the banana rachis, sisal, kapok, pineapple leaf and coir respectively.

The degradation temperature of the bleached cellulose fibers was appreciably increased compared to that of the original untreated samples. The higher temperatures of thermal decomposition of the bleached cellulose fibers are related to the partial removal of hemicelluloses, lignin, and pectins, which degrade at lower temperatures. The acid hydrolysis followed by mechanical homogenization had significant effect on the thermal stability of the nanocellulose. The DTG curves of extracted nanocellulose showed a two-stage degradation pattern. The initial degradation around $100{ }^{\circ} \mathrm{C}$ corresponds to the loss of moisture evaporation and the second degradation 
Fig. 10 TG and DTG curves of $a$ untreated plant fibers, $b$ bleached cellulose fibers and $c$ nanocellulose of A banana rachis $\mathbf{B}$ sisal C kapok D pineapple leaf and $\mathbf{E}$ coir
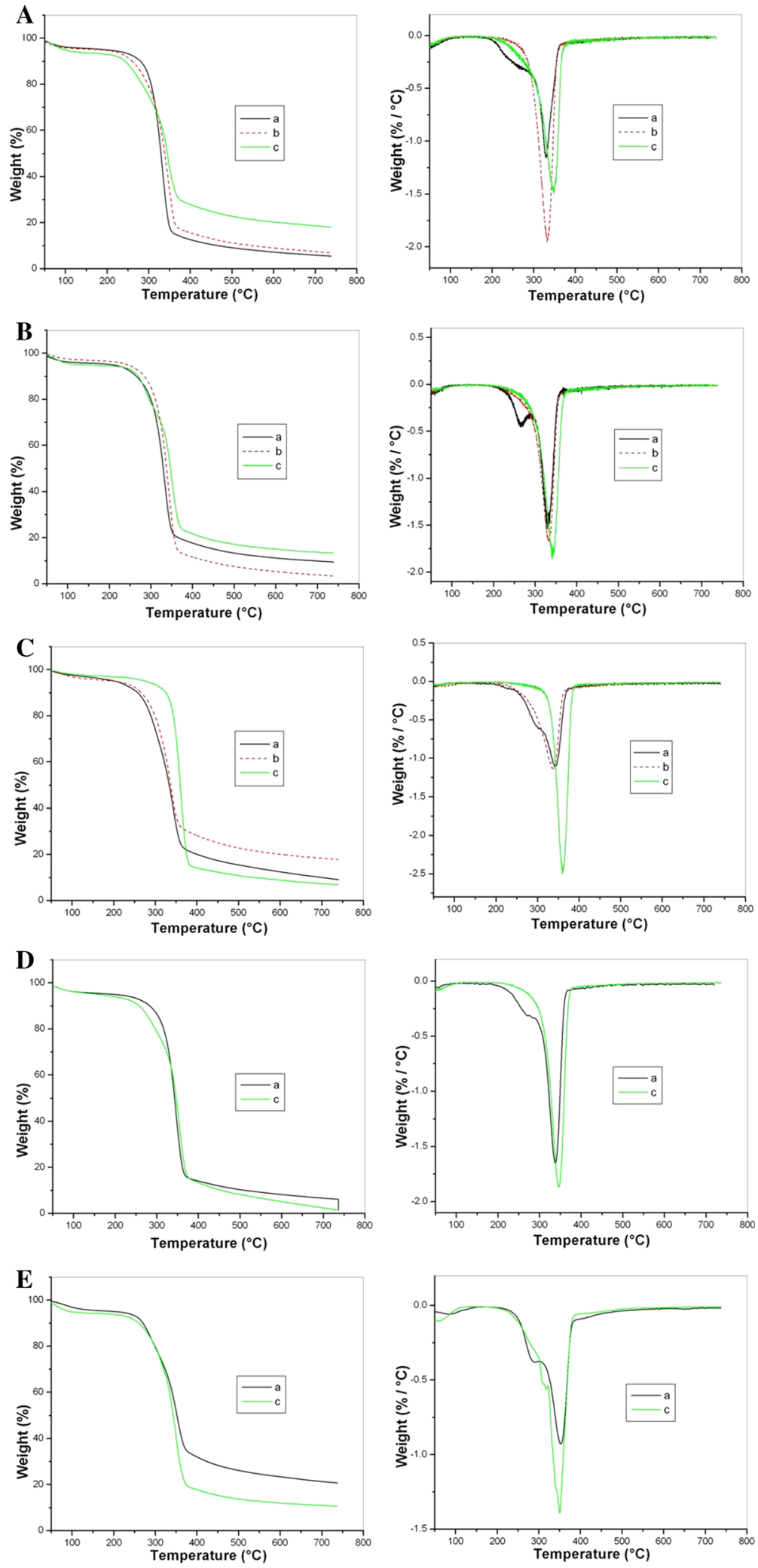
Table 4 TGA results of untreated plant fibers, bleached cellulose fibers and the isolated nanocellulose from banana rachis, sisal, kapok, pineapple leaf and coir

\begin{tabular}{lllc}
\hline Sample & $\begin{array}{l}\text { Cellulose } \\
\text { decomposition } \\
\text { temperature }\left({ }^{\circ} \mathrm{C}\right)\end{array}$ & $\begin{array}{l}\text { Weight } \\
\text { loss }(\%)^{\mathrm{a}}\end{array}$ & $\begin{array}{c}\text { Residual } \\
\text { char }(\%)^{\mathrm{b}}\end{array}$ \\
\hline RB & 329.8 & 54.7 & 18.1 \\
BB & 346.4 & 52.5 & 6.8 \\
BNC & 351.3 & 68.7 & 5.5 \\
RS & 331.0 & 55.1 & 13.3 \\
BS & 337.4 & 59.3 & 9.5 \\
SNC & 347.9 & 53.1 & 3.4 \\
RK & 346.4 & 64.4 & 17.8 \\
BK & 341.5 & 54.5 & 9.1 \\
KNC & 360.4 & 56.9 & 11.3 \\
RP & 337.1 & 48.1 & 6.9 \\
PNC & 348.7 & 62.6 & 6.1 \\
RC & 353.3 & 51.4 & 20.7 \\
CNC & 348.7 & 58.5 & 10.6 \\
\hline
\end{tabular}

$R B$ raw banana rachis, $\mathrm{BB}$ bleached banana rachis, $B N C$ banana rachis nanocellulose, $R S$ raw sisal, $B S$ bleached sisal, $S N C$ sisal nanocellulose, $R K$ raw kapok, $B K$ bleached kapok, $K N C$ kapok nanocellulose, $R P$ raw pineapple leaf, $P N C$ pineapple leaf nanocellulose, $R C$ raw coir, $C N C$ coir nanocellulose

a at the cellulose decomposition temperature

b at $700{ }^{\circ} \mathrm{C}$

around $310-380{ }^{\circ} \mathrm{C}$ attributed to cellulose decomposition. The acid hydrolyzed specimens of isolated nanocellulose from all the fiber sources except the coir fiber showed a further improvement in thermal stability. The nanocellulose extracted from coir fiber showed a slight reduction in thermal stability than the cellulose present in the untreated coir fibers. This is due to the reduced strength of lignin-cellulose complex in the nanocellulose compared to that of the untreated coir fiber. Raw coir fiber has a lignin content of $45-50 \%$ of weight. In the raw coir fiber, the cellulose and lignin are packed as a strong lignincellulose complex which is more thermally stable than pure cellulose.

The degradation temperatures of the isolated nanocellulose of banana rachis, sisal, kapok, pineapple leaf and coir were found to be around 351, 348, 360, 347 and $348{ }^{\circ} \mathrm{C}$, respectively (Table 4). The small variation (around $1.6 \%$ ) indicated that the obtained nanocelluloses are similar in terms of the composition and consequently in the thermal stability. The higher thermal stability of the nanocellulose can be attributed to the tangling effect of flexible nanocellulose (Maiti et al. 2013) and hemicelluloses and lignin removal from these fibers and higher crystallinity of the cellulose (Alemdar and Sain 2008; Sain and Panthapulakkal 2006). These results are very consistent with the results obtained from FTIR and XRD measurements. The higher thermal stability of the isolated nanocellulose from various sources promotes their effective utilization as reinforcing agent in the production of bionanocomposites.

\section{Conclusions}

In the present study, nanocellulose was successfully isolated from various plant fiber sources (banana rachis, sisal, kapok, pineapple leaf and coir) using acid hydrolysis coupled with steam explosion process. The morphology, crystallinity, birefringence, thermal and surface properties of isolated nanocelluloses were analyzed and compared using different characterization techniques. FTIR measurements on these fibers revealed the removal of hemicelluloses and lignin during the chemical process. Morphological analysis by TEM supports the evidence for the successful isolation of cellulose nanofibrils with an average diameter of 10-25 nm. XRD results confirmed the highly crystalline nature of cellulose nanofibrils isolated. IGC results showed that the obtained nanocelluloses have similar surface areas and an amphoteric or a predominantly basic surface. TGA and DTG results revealed the higher thermal stability of the isolated nanocellulose than the corresponding raw materials. This study promotes the effective utilization of lignocellulosic fibers as a renewable source of nanocellulose for the production of bionanomaterials for diversified applications.

Acknowledgments L.A. Pothan is grateful to the University Grants Commission (UGC), Government of India, for the Emeritus fellowship.

\section{References}

Abdul Khalil HPS, Davoudpour Y, Islam MN, Mustapha A, Sudesh K, Dungani R, Jawaid M (2014) Production and modification of nanofibrillated cellulose using various mechanical processes: a rewiew. Carbohydr Polym 99:649-665

Abraham E, Deepa B, Pothan LA, Jacob M, Thomas S, Anandjiwala R (2011) Extraction of nanocellulose fibrils from 
lignocellulosic fibers: a novel approach. Carbohydr Polym 86:1468-1475

Alemdar A, Sain M (2008) Isolation and characterization of nanofibers from agricultural residues-wheat straw and soy hulls. Bioresour Technol 99:1664-1671

Chakraborty A, Sain M, Kortschot M (2005) Cellulose microfibrils: a novel method of preparation using high shear refining and cryocrushing. Holzforschung 59:102-107

Chen WS, Yu HP, Liu YX, Chen P, Zhang MX, Hai YF (2011) Individualization of cellulose nanofibers from wood using high-intensity ultrasonication combined with chemical pretreatments. Carbohydr Polym 83:1804-1811

Chirayil CJ, Joy J, Mathew L, Mozetic M, Koetz J, Thomas S (2014) Isolation and characterization of cellulose nanofibrils from Helicteresisora plant. Ind Crop Prod 59:27-34

Cordeiro N, Gouveia C, Moraes AGO, Amico SC (2011) Natural fibers characterization by inverse gas chromatography. Carbohydr Polym 84:110-117

Cordeiro N, Mendonça C, Pothan LA, Varma A (2012) Monitoring surface properties evolution of thermochemically modified cellulose nanofibers from banana pseudo-stem. Carbohydr Polym 88:125-131

Das K, Ray D, Bandyopadhyay NR, Sahoo S, Mohanty AK, Misra M (2011) Physicomechanical properties of the jute micro/nanofibril reinforced starch/polyvinyl alcohol biocomposite films. Compos Part B 42:376-381

Deng H, Zhou X, Wang X, Zhang C, Ding B, Zhang Q, Du Y (2010) Layer-by layer structured polysaccharides filmcoated cellulose nanofibrous mats for cell culture. Carbohydr Polym 80:475-480

Eichhorn SJ (2011) Cellulose nanowhiskers: promising materials for advanced applications. Soft Matter 7:303-315

Ferrer A, Filpponen I, Rodríguez A, Laine J, Rojas OJ (2012) Valorization of residual Empty Palm Fruit Bunch Fibers (EPFBF) by microfluidization: production of nanofibrillated cellulose and EPFBF nanopaper. Bioresour Technol 125:249-255

Fortunati E, Puglia D, Monti M, Peponi L, Santulli C, Kenny JM, Torre L (2013) Extraction of cellulose nanocrystals from phormium tenax fibers. J Polym Environ 21:319-328

Fukuzumi H, Saito T, Isogai A (2013) Influence of TEMPOoxidized cellulose nanofibril length on film properties. Carbohydr Polym 93:172-177

Habibi Y, Lucia LA, Rojas OJ (2010) Cellulose nanocrystals: chemistry, self-assembly, and applications. Chem Rev 110:3479-3500

Hossain KMZ, Hasan MS, Boyd D, Rudd CD, Ahmed I, Thielemans W (2014) Effect of cellulose nanowhiskers on surface morphology, mechanical properties, and cell adhesion of melt-drawn polylactic acid fibers. Biomacromolecules 15:1498-1506

Isogai T, Saito T, Isogai A (2011) Wood cellulose nanofibrils prepared by TEMPO electro-mediated oxidation. Cellulose 18:421-431

Jiang F, Hsieh Y-L (2013) Chemically and mechanically isolated nanocellulose and their self-assembled structures. Carbohydr Polym 95:32-40

Jiang F, Han S, Hsieh Y-L (2013) Controlled defibrillation of rice straw cellulose and self-assembly of cellulose nanofibrils into highly crystalline fibrous materials. RSC Adv $3: 12366-12375$
Kaushik A, Singh M (2011) Isolation and characterization of cellulose nanofibrils from wheat straw using steam explosion coupled with high shear homogenization. Carbohydr Res 346:76-85

Kengkhetkit N, Amornsakchai T (2014) A new approach to "Greening" plastic composites using pineapple leaf waste for performance and cost effectiveness. Mater Des 55:292-299

Klemm D, Kramer F, Moritz S, Lindstrom T, Ankerfors M, Gray D, Dorris A (2011) Nanocelluloses: a new family of naturebased materials. Angew Chem Int Ed 50:5438-5466

Kumar A, Negi YS, Choudhary V, Bhardwaj NK (2014) Characterization of cellulose nanocrystals produced by acid-hydrolysis from sugarcane bagasse as agro-waste. J Mater Phys Chem 2:1-8

Lin J, Tian F, Zhao N, Li X, Bian F, Wang J (2014) Cellulose nanofibrils aerogels generated from jute fibers. Carbohydr Polym 109:35-43

Maiti S, Jayaramudu J, Das K, Reddy SM, Sadiku R, Ray SS, Liu D (2013) Preparation and characterization of nanocellulose with new shape from different precursor. Carbohyd Polym 98:562-567

Mandal A, Chakrabarty D (2011) Isolation of nanocellulose from waste sugarcane bagasse (SCB) and its characterization. Carbohydr Polym 86:1291-1299

Mathew AP, Oksman K, Karim Z, Liu P, Khan SA, Naseri N (2014) Process scale up and characterization of wood cellulose nanocrystals hydrolysed using bioethanol pilot plant. Ind Crop Prod 58:212-219

Missoum K, Belgacem M, Bras J (2013) Nanofibrillated cellulose surface modification: a review. Materials 6:1745-1766

Moon RJ, Martini A, Nairn J, Simonsen J, Youngblood J (2011) Cellulose nanomaterials review: structure, properties and nanocomposites. Chem Soc Rev 40:3941-3994

Moran JI, Alvarez VA, Cyras VP, Vazquez A (2008) Extraction of cellulose and preparation of nanocellulose from sisal fibers. Cellulose 15:149-159

Nakagaito AN, Yano H (2004) The effect of morphological changes from pulp fiber towards nano-scale fibrillated cellulose on the mechanical properties of high strength plant fiber based composites. Appl Phys A Mater 78:547-552

Nishiyama Y, Sugiyama J, Chanzy H, Langan P (2003) Crystal structure and hydrogen bonding system in cellulose $\mathrm{I} \alpha$ from synchrotron X-ray and neutron fiber diffraction. J Am Chem Soc 125:14300-14306

Panthapulakkal S, Sain M (2012) Preparation and characterization of cellulose nanofibril films from wood fiber and their thermoplastic polycarbonate composites. Int J Polym Sci 2012:1-6

Sacui IA, Nieuwendaal RC, Burnett DJ, Stranick SJ, Jorfi M, Weder C, Foster EJ, Olsson RT, Gilman JW (2014) Comparison of the properties of cellulose nanocrystals and cellulose nanofibrils isolated from bacteria, tunicate, and wood processed using acid, enzymatic, mechanical, and oxidative methods. ACS Appl Mater Interfaces 6:6127-6138

Sain M, Panthapulakkal S (2006) Bioprocess preparation of wheat straw fibers and their characterization. Ind Crop Prod 23:1-8

Saito T, Uematsu T, Kimura S, Enomae T, Isogai A (2011) Selfaligned integration of native cellulose nanofibrils towards producing diverse bulk materials. Soft Matter 7:8804-8809

Segal L, Creely JJ, Martin AE, Conrad CM (1959) An empirical method for estimating the degree of crystallinity of native 
cellulose using the X-ray diffractometer. Text Res J 29:786-794

Silvério HA, Flauzino Neto WP, Dantas NO, Pasquini D (2013) Extraction and characterization of cellulose nanocrystals from corncob for application as reinforcing agent in nanocomposites. Ind Crop Prod 44:427-436

Stenstad P, Andresen M, Tanem BS, Stenius P (2008) Chemical surface modification of microfibrillated cellulose. Cellulose 15:35-45

Wang QQ, Zhu JY, Gleisner R, Kuster TA, Baxa U, McNeil SE (2012) Morphological development of cellulose fbrils of a bleached eucalyptus pulp by mechanical fibrillation. Cellulose 19:1631-1643

Wicklein B, Salazar-Alvarez G (2013) Functional hybrids based on biogenic nanofibrils and inorganic nanomaterials. J Mater Chem A 1:5469-5478

Xu X, Liu F, Jiang L, Zhu JY, Haagenson D, Wiesenborn DP (2013) Cellulose nanocrystals vs cellulose nanofibrils: a comparative study on their microstructures and effects as polymer reinforcing agents. ACS Appl Mater Interfaces 5:2999-3009 